CUBO A Mathematical Journal

Vol.16, Noㅡ, (111-119). June 2014

\title{
On the uniform asymptotic stability to certain first order neutral differential equations
}

\author{
Cemil TunÇ \\ Department of Mathematics, \\ Faculty of Science, \\ Yüzüncü Yıl University, 65080, Van, Turkey \\ cemtunc@yahoo.com
}

\begin{abstract}
In this paper, the uniform asymptotic stability of the zero solution of a kind of neutral differential equations is discussed. Based on the Lyapunov functional approach, a new stability criterion is derived, which is delay dependent on two positive constants. The result to be obtained here extends and generalizes the existing ones in the literature.
\end{abstract}

\section{RESUMEN}

En este artículo se discute la estabilidad asintótica uniforme de la solución cero de un tipo de ecuaciones diferenciales neutrales. Basados en la técnica de funcional de Lyapunov, se deriva un nuevo criterio de estabilidad, el cual es dependiente de atrasos basados en dos constantes positivas. El resultado que se obtiene extiende y generaliza los encontrados en la literatura.

Keywords and Phrases: Neutral differential equation; first order, uniform asymptotic stability; Lyapunov functional.

2010 AMS Mathematics Subject Classification: 34K20, 34K40. 


\section{Introduction}

In 2009, Nam and Phat [4] considered the first order neutral differential equation

$$
\frac{d}{d t}[x(t)+p x(t-\tau)]=-a x(t)+b \tanh x(t-\sigma), t \geq 0
$$

where $a, \tau$ and $\sigma$ are positive constants, $\sigma \geq \tau, b$ and $p$ are real numbers with $|p|<1$. Using a Lyapunov functional, the authors established some sufficient conditions for the zero solution of Eq. (1.1) to be uniformly asymptotically stable. By this work, Nam and Phat [4] established an improved criterion for the uniform asymptotic stability of the zero solution of Eq. (1.1).

In this paper, instead of Eq. (1.1), we consider the first order neutral differential equation of the form

$$
\frac{d}{d t}[x(t)+p x(t-\tau)]=-f(x(t)) x(t)-g(x(t-\tau)) x(t)+b \tanh x(t-\sigma), t \geq 0
$$

where $f$ and $g$ are continuous functions on $\mathfrak{R}=(-\infty, \infty), \tau$ and $\sigma$ are positive constants with $\sigma \geq \tau, b$ and $p$ are real numbers, $|p|<1$. For each solution of Eq. (1.2), we assume the initial condition

$$
x(t)=\phi(t), t \in[-\sigma, 0], \phi \in C([-\sigma, 0], \mathfrak{R})
$$

A primary purpose of this paper is to study the uniform asymptotic stability of the zero solution of Eq. (1.2). Motivated by Nam and Phat [4], we obtain some sufficient conditions which guarantee the uniform asymptotic stability of the zero solution of Eq. (1.2). It is clear that the equation discussed by Nam and Phat [4], Eq. (1.1), is a special case of our equation, Eq. (1.2). That is, our equation, Eq. (1.2), includes and extends the equation discussed in [4]. By this paper, we generalize and extend the result obtained by Nam and Phat [4].

Finally, in paticular, one can refer to the papers of Agarwal and Grace [1], Park [5], Sun and Wang [6], Tunç [7], Tunç and Sirma [9] and the references listed in these sources to see some recent contributions focused on the topic of this paper.

\section{Description of problem}

Before introducing our main result, we give some basic information relative to the topic.

Lemma 2.1. ([4]). Assume that $\mathrm{S} \in \mathfrak{R}^{\mathrm{n} \times \mathfrak{n}}$ is a symmetric positive definite matrix. Then for every $\mathrm{Q} \in \mathfrak{R}^{\mathrm{n} \times \mathrm{n}}$,

$$
2<\mathrm{Qy}, x>\leq<\mathrm{QS}^{-1} \mathrm{Q}^{\top} \mathrm{x}, \mathrm{x}>+<\mathrm{Sy}, \mathrm{y}>, \forall x, \mathrm{y} \in \mathfrak{R}^{\mathrm{n}}
$$


Lemma 2.2. ([2]). For any symmetric positive definite matrix $M \in \mathfrak{R}^{\mathfrak{n} \times \mathfrak{n}}$, scalar $\sigma \geq 0$ and vector function $w:[0, \sigma] \rightarrow \mathfrak{R}^{\mathfrak{n}}$ such that the integrations concerned are well defined, then

$$
\left(\int_{0}^{\sigma} w(s) d s\right)^{\top} M\left(\int_{0}^{\sigma} w(s) d s\right) \leq \sigma \int_{0}^{\sigma} w^{\top}(s) M w(s) d s .
$$

Definition ([3]). Suppose $\Omega \subseteq \mathfrak{R} \times C$ is open, $f: \Omega \rightarrow \mathfrak{R}^{\mathfrak{n}}, \mathrm{D}: \Omega \rightarrow \mathfrak{R}^{\mathfrak{n}}$ are given continuous functions with $\mathrm{D}$ atomic at zero (see Hale and Lunel [[3], pp.53]. The relation

$$
\frac{d}{d t} D\left(t, x_{t}\right)=f\left(t, x_{t}\right)
$$

is called the neutral functional differential equation.

Theorem 2.1. ([3]). Suppose D is a stable operator, $f: \mathfrak{R} \times \mathbb{C} \rightarrow \mathfrak{R}^{n}, f: \mathfrak{R} \times$ (bounded sets of C) into bounded sets of $\mathfrak{R}^{n}$ and suppose $\mathrm{u}(\mathrm{s}), \boldsymbol{v}(\mathrm{s})$, and $\boldsymbol{w}(\mathrm{s})$ are continuous, nonnegative, and non-decreasing with $\mathrm{u}(\mathrm{s}), \boldsymbol{v}(\mathrm{s})>0$ for $\mathrm{s} \neq 0$, and $\mathrm{u}(0)=v(0)=0$. If there is a continuous function $\mathrm{V}: \mathfrak{R} \times \mathrm{C} \rightarrow \mathfrak{R}^{\mathrm{n}}$ such that

$$
\mathrm{u}(|\mathrm{D} \phi|) \leq \mathrm{V}(\mathrm{t}, \phi) \leq v(|\phi|)
$$

and

$$
\dot{\mathrm{V}}(\mathrm{t}, \phi) \leq-w(|\mathrm{D} \phi|),
$$

then the solution $\mathrm{x}=0$ of $\frac{\mathrm{d}}{\mathrm{dt}} \mathrm{D}\left(\mathrm{t}, \mathrm{x}_{\mathrm{t}}\right)=\mathrm{f}\left(\mathrm{t}, \mathrm{x}_{\mathrm{t}}\right)$ are uniformly stable. If $\mathrm{u}(\mathrm{s}) \rightarrow \infty$ as $\mathrm{s} \rightarrow \infty$, then the solutions of $\frac{\mathrm{d}}{\mathrm{dt}} \mathrm{D}\left(\mathrm{t}, \mathrm{x}_{\mathrm{t}}\right)=\mathrm{f}\left(\mathrm{t}, \mathrm{x}_{\mathrm{t}}\right)$ are uniformly bounded. If $\mathrm{w}(\mathrm{s})>0$ for $\mathrm{s}>0$, then the solution $\mathrm{x}=0$ of $\frac{\mathrm{d}}{\mathrm{dt}} \mathrm{D}\left(\mathrm{t}, \mathrm{x}_{\mathrm{t}}\right)=\mathrm{f}\left(\mathrm{t}, \mathrm{x}_{\mathrm{t}}\right)$ is uniformly asymptotically stable. The same conclusion holds if the upper bound of $\dot{\mathrm{V}}(\mathrm{t}, \phi)$ is given by $-\mathfrak{w}(|\phi(0)|$.

For a real number $\alpha$, Eq. (1.2) can be rewritten in the form

$$
\begin{aligned}
& \frac{d}{d t}\left[x(t)+p x(t-\tau)+\alpha \int_{t-\tau}^{t} x(s) d s+b \int_{t-\sigma}^{t-\tau} \tanh x(s) d s\right] \\
= & \{\alpha-[f(x(t))+g(x(t-\tau))]\} x(t)-\alpha x(t-\tau)+b \tanh x(t-\tau), t \geq 0 .
\end{aligned}
$$

Define the operators $([4])$ :

$$
D_{1}\left(x_{t}\right)=x(t)+p x(t-\tau)+\alpha \int_{t-\tau}^{t} x(s) d s+b \int_{t-\sigma}^{t-\tau} \tanh x(s) d s
$$

and

$$
D_{2}\left(x_{t}\right)=x(t)+p x(t-\tau) .
$$

Our main result is the following theorem. 
Theorem 2.2. In addition to the all the basic assumptions imposed to the functions $\mathrm{f}$ and $\mathrm{g}$ that appear in Eq. (1.2), we assume that there exist a constant $\alpha$ with $0<|\alpha|<1$ and positive constants $\beta, \gamma, \eta, \theta, a_{1}$ and $a_{2}$ such that the following conditions hold:

$$
\Omega=\left[\begin{array}{ccccc}
\Omega_{11} & \Omega_{12} & b & \Omega_{14} & \Omega_{15} \\
* & -\beta-2 p \alpha & p b & -\alpha & -b \alpha \\
* & * & \theta(\sigma-\tau)^{2}-\eta & b & b^{2} \\
* & * & * & -\gamma & 0 \\
* & * & * & * & -\theta
\end{array}\right]<0,
$$

where

$$
\begin{gathered}
\Omega_{11}=2(\alpha-a)+\beta+\gamma \tau^{2}+\eta, \\
\Omega_{12}=p(\alpha-f(x)-g(x(t-\tau)))-\alpha, \\
\Omega_{14}=\alpha-f(x(t))-g(x(t-\tau)), \\
\Omega_{15}=b\{\alpha-f(x(t))-g(x(t-\tau))\}
\end{gathered}
$$

Then, the zero solution of Eq. (1.2) is uniformly asymptotically stable.

Remark. Here the symbol * represents the elements below the main diagonal of the matrix $\Omega$ in (4).

Proof. Since $\Omega<0$, there is a positive constant $\delta$ such that the following inequality holds:

$$
\Omega_{1}=\left[\begin{array}{ccccc}
\Omega_{11} & \Omega_{12} & \mathrm{~b} & \Omega_{14} & \Omega_{15} \\
* & -\beta-2 p \alpha & \mathrm{pb} & -\alpha & -\mathrm{b} \alpha \\
* & * & \theta(\sigma-\tau)^{2}-\eta+\delta & \mathrm{b} & \mathrm{b}^{2} \\
* & * & * & -\gamma & 0 \\
* & * & * & * & -\theta
\end{array}\right]<0 .
$$

We use the Lyapunov functional ([4]) defined by

$$
\mathrm{V}=\mathrm{V}_{1}+\mathrm{V}_{2}+\mathrm{V}_{3}+\mathrm{V}_{4}+\mathrm{V}_{5}+\mathrm{V}_{6}+\mathrm{V}_{7}
$$


where

$$
\begin{gathered}
V_{1}=D_{1}^{\top}\left(x_{t}\right) D_{1}\left(x_{t}\right), \\
V_{2}=\beta \int_{t-\tau}^{t} x^{2}(s) d s, \\
V_{3}=\gamma \tau \int_{t-\tau}^{t}(\tau-t+s)(\alpha x(s))^{2} d s, \\
V_{4}=\theta(\sigma-\tau) \int_{t-\sigma}^{t-\tau}(s-t+\sigma) \tanh ^{2} x(s) d s, \\
V_{6}=\delta \int_{t-\sigma}^{t-\tau} \tanh ^{2} x(s) d s,(\delta \text { is a positive constant }), \\
V_{5}=\eta \int_{t-\tau}^{t} \tanh ^{2} x(s) d s, \\
V_{7}=\varepsilon D_{2}^{2}\left(x_{t}\right),
\end{gathered}
$$

$\varepsilon$ is a positive constant that will be chosen later.

Let $V_{0}=V_{1}+V_{2}+V_{3}+V_{4}+V_{5}+V_{6}$. The time derivative of the functional $V_{0}$ along solutions of Eq. (2.1) is given by

$$
\begin{aligned}
\frac{d}{d t} V_{0}= & 2\left[x(t)+p x(t-\tau)+\alpha \int_{t-\tau}^{t} x(s) d s+b \int_{t-\sigma}^{t-\tau} \tanh x(s) d s\right]^{T} \\
& \times[\{\alpha-[f(x(t))+g(x(t-\tau))]\} x(t)-\alpha x(t-\tau)+b \tanh x(t-\tau)] \\
& +\beta x^{2}(t)-\beta x^{2}(t-\tau)+\gamma \tau^{2} \alpha^{2} x^{2}(t)-\gamma \tau \int_{t-\tau}^{t}(\alpha x(s))^{2} d s \\
& +\theta(\sigma-\tau)^{2} \tanh ^{2} x(t-\tau)-\theta(\sigma-\tau) \int_{t-\sigma}^{t-\tau} \tanh ^{2} x(s) d s \\
& +\eta \tanh ^{2} x(t)-\eta \tanh ^{2} x(t-\tau)+\delta \tanh ^{2} x(t-\tau)-\delta \tanh ^{2} x(t-\sigma) .
\end{aligned}
$$


Using Lemma 2.2 and applying the estimate $\tanh ^{2} x(t) \leq \chi^{2}(t)$, we obtain

$$
\begin{aligned}
\frac{d}{d t} V_{0} \leq & 2\left[x(t)+p x(t-\tau)+\alpha \int_{t-\tau}^{t} x(s) d s+b \int_{t-\sigma}^{t-\tau} \tanh x(s) d s\right]^{T} \\
& \times[\{\alpha-[f(x(t))+g(x(t-\tau)]] x x(t)-\alpha x(t-\tau)+b \tanh x(t-\tau)] \\
& +\beta x^{2}(t)-\beta x^{2}(t-\tau)+\gamma \tau^{2} \alpha^{2} x^{2}(t)-\gamma\left(\int_{t-\tau}^{t} \alpha x(s) d s\right)^{2} \\
& +\theta(\sigma-\tau)^{2} \tanh ^{2} x(t-\tau)-\theta\left(\int_{t-\sigma}^{t-\tau} \tanh x(s) d s\right)^{2} \\
& +\eta x^{2}(t)-\eta \tanh ^{2} x(t-\tau)+\delta \tanh ^{2} x(t-\tau)-\delta \tanh ^{2} x(t-\sigma) .
\end{aligned}
$$

Choosing

$$
\alpha \int_{\mathrm{t}-\tau}^{\mathrm{t}} x(\mathrm{~s}) \mathrm{d} s=\mathrm{u}(\mathrm{t}), \int_{\mathrm{t}-\sigma}^{\mathrm{t}-\tau} \tanh x(\mathrm{~s}) \mathrm{d} s=v(\mathrm{t})
$$

and using the assumptions $f(x) \geq a_{1}, g(x) \geq a_{2}$ and the estimate $0<|\alpha|<1$, it follows that

$$
\begin{aligned}
\frac{d}{d t} V_{0} \leq & {\left[2\left\{\alpha-\left(a_{1}+a_{2}\right)\right\}+\beta+\gamma \tau^{2}+\eta\right] x^{2}(t) } \\
& +[-2 \alpha+2 p(\alpha-f(x(t))-g(x(t-\tau)))] x(t) x(t-\tau) \\
& +2 b x(t) \tanh x(t-\tau)+2\{\alpha-f(x(t))-g(x(t-\tau))\} x(t) u(t) \\
& +2 b\{\alpha-f(x(t))-g(x(t-\tau))\} x(t) v(t) \\
& -\left(2 p \alpha+\beta^{2}\right) x^{2}(t-\tau)+2 p b x(t-\tau) \tanh x(t-\tau)-2 \alpha x(t-\tau) u(t) \\
& -2 \alpha b x(t-\tau) v(t)+\left[\theta(\sigma-\tau)^{2}-\eta+\delta\right] \tanh ^{2} x(t-\tau)-\delta \tanh ^{2} x(t-\sigma) \\
& +2 b \tanh x(t-\tau) u(t)+2 b^{2} \tanh x(t-\tau) v(t)-\gamma u^{2}(t)-\theta v^{2}(t) \\
= & {\left[2(\alpha-a)+\beta+\gamma \tau^{2}+\eta\right] x^{2}(t) } \\
& +[-2 \alpha+2 p(\alpha-f(x(t))-g(x(t-\tau)))] x(t) x(t-\tau) \\
& +2 b x(t) \tanh x(t-\tau)+2(\alpha-f(x(t))-g(x(t-\tau))) x(t) u(t) \\
& +2 b(\alpha-f(x(t))-g(x(t-\tau))) x(t) v(t) \\
& -\left(2 p \alpha+\beta^{2}\right) x^{2}(t-\tau)+2 p b x(t-\tau) \tanh x(t-\tau)-2 \alpha x(t-\tau) u(t) \\
& -2 \alpha b x(t-\tau) v(t)+\left[\theta(\sigma-\tau)^{2}-\eta+\delta\right] \tanh ^{2} x(t-\tau)-\delta \tanh ^{2} x(t-\sigma) \\
& +2 b \tanh x(t-\tau) u(t)+2 b^{2} \tanh x(t-\tau) v(t)-\gamma u^{2}(t)-\theta v^{2}(t) .
\end{aligned}
$$

Since the terms on the right hand side of $\frac{\mathrm{d}}{\mathrm{dt}} \mathrm{V}_{0}$ represent a specific quadratic form, we can rearrange the terms given on the right hand side of $\frac{d}{d t} V_{0}$ as the following:

$$
\frac{d}{d t} V_{0} \leq \zeta^{\top}(t) \Omega_{2} \zeta(t)
$$


where

$$
\begin{gathered}
\Omega_{2}=\left[\begin{array}{cccccc}
\Omega_{11} & \Omega_{12} & b & \Omega_{14} & \Omega_{15} & 0 \\
* & -\beta-2 p \alpha & p b & -\alpha & -b \alpha & 0 \\
* & * & \theta(\sigma-\tau)^{2}-\eta+\delta & b & b^{2} & 0 \\
* & * & * & -\gamma & 0 & 0 \\
* & * & * & * & -\theta & 0 \\
* & * & * & * & * & -\delta
\end{array}\right] . \\
\zeta^{\top}(t)=\left[\begin{array}{c}
x(t) \\
x(t-\tau) \\
\tanh x(t-\tau) \\
u(t) \\
v(t)
\end{array}\right]^{\top}, \zeta(t)=\left[\begin{array}{c}
x(t-\tau) \\
\tanh x(t-\tau) \\
u(t) \\
v(t) \\
\tanh x(t-\sigma)
\end{array}\right] .
\end{gathered}
$$

Making use of the assumption $\Omega<0$, we have

$$
\frac{d}{d t} V_{0} \leq \zeta^{\top}(t) \Omega_{2} \zeta(t)<0 .
$$

Therefore, there exists a positive constant $\lambda$ such that

$$
\begin{aligned}
\frac{d}{d t} V_{0} \leq & -\lambda\left\{\|x(t)\|^{2}+\|x(t-\tau)\|^{2}+\|\tanh x(t-\tau)\|^{2}\right. \\
& \left.+\|u(t)\|^{2}+\|v(t)\|^{2}+\|\tanh x(t-\sigma)\|^{2}\right\} .
\end{aligned}
$$

By a straightforward calculation for the time derivative of $V_{7}$, it follows that

$$
\begin{aligned}
\frac{d}{d t} V_{7}= & 2 \varepsilon[x(t)+p x(t-\tau)][-f(x(t)) x(t)-g(x(t-\tau)) x(t)+b \tanh x(t-\sigma)] \\
= & -2 \varepsilon f(x(t)) x^{2}(t)-2 \varepsilon g(x(t-\sigma)) x^{2}(t)+2 b \varepsilon x(t) \tanh x(t-\sigma) \\
& -2 \varepsilon p f(x(t)) x(t) x(t-\tau)-2 \varepsilon p g(x(t-\tau)) x(t) x(t-\tau) \\
& +2 \varepsilon p b x(t-\tau)) \tanh x(t-\sigma)
\end{aligned}
$$

Taking into account the assumptions $f(x) \geq a_{1}, g(x) \geq a_{2}, a=a_{1}+a_{2}$, and Lemma 2.1, we find

$$
\begin{aligned}
\frac{d}{d t} V_{7} \leq & -2 \varepsilon\left(a_{1}+a_{2}\right) x^{2}(t)+2 b \varepsilon x(t) \tanh x(t-\sigma) \\
& +2 \varepsilon p\{f(x(t))+g(x(t-\tau))\}|x(t)||x(t-\tau)| \\
& +2 \varepsilon p b x(t-\tau)) \tanh x(t-\sigma) \\
\leq & -2 \varepsilon a x^{2}(t)+2 b \varepsilon x(t) \tanh x(t-\sigma)+2 \varepsilon a p|x(t)||x(t-\tau)| \\
& +2 \varepsilon p b x(t-\tau)) \tanh x(t-\sigma) \\
\leq & \varepsilon\left\{-2 a+|b|^{2}+|a p|^{2}\right\} x^{2}(t)+\varepsilon\left\{1+|b p|^{2}\right\} x^{2}(t-\tau)+2 \varepsilon \tanh ^{2} x(t-\sigma) .
\end{aligned}
$$


Let us choose the constant $\varepsilon$ as

$$
\varepsilon=\left\{\begin{array}{l}
2^{-1} \lambda \min \left\{\left(1+|b p|^{2}\right)^{-1}, 2^{-1}\right\}, \text { if }-2 a+|b|^{2}+|a p|^{2} \leq 0 \\
2^{-1} \lambda \min \left\{\left(1+|b|^{2}+|a p|^{2}\right)^{-1},\left(1+|b p|^{2}\right)^{-1}, 2^{-1}\right\}, \quad \text { if }-2 a+|b|^{2}+|a p|^{2}>0 .
\end{array}\right.
$$

Hence, we can obtain

$$
\frac{d}{d t} V<-\frac{\lambda}{2}\|x(t)\|^{2}
$$

Further, we have $\mathrm{V} \geq \varepsilon \mathrm{D}_{2}^{2}\left(\mathrm{x}_{\mathrm{t}}\right)$, and $\mathrm{D}_{2}\left(\mathrm{x}_{\mathrm{t}}\right)$ is stable due to $|\mathrm{p}|<1$.

The proof of Theorem (2.2) is now completed.

\section{Conclusion}

A non-linear neutral differential equation of the first order is considered. We establish certain sufficient conditions which guarantee that the zero solution of this equation is uniformly asymptotically stable. In proving our main result, we employ the Lyapunov functional approach as a basic tool. This paper has a contribution to the subject in the literature, and it may be useful for researchers work on the qualitative behaviors of solutions.

\section{Received: December 2012. Revised: May 2013.}

\section{References}

[1] Agarwal, R. P.; Grace, S. R., Asymptotic stability of certain neutral differential equations, Math. Comput. Modelling, 31 (2000), no. 8-9, 9-15.

[2] Gu, K., An integral inequality in the stability problem of time-delay system systems. In: Proceedings of 39th IEEE CDC, Sydney, Australia, 2000, pp. 2805-2810.

[3] Hale, Jack K.; Verduyn Lunel, Sjoerd M., Introduction to functional-differential equations. Applied Mathematical Sciences, 99. Springer-Verlag, New York, 1993.

[4] Nam, P. T.; Phat, V. N., An improved stability criterion for a class of neutral differential equations, Appl. Math.Lett., 22 (2009), no. 1, 31-35.

[5] Park, J. H., Delay-dependent criterion for asymptotic stability of a class of neutral equations, Appl. Math. Lett., 17 (2004), no. 10, 1203-1206.

[6] Sun, Y. G.; Wang, L., Note on asymptotic stability of a class of neutral differential equations, Appl. Math. Lett., 19 (2006), no. 9, 949-953.

[7] Tunç, C., Asymptotic stability of nonlinear neutral differential equations with constant delays: a descriptor system approach, Ann. Differential Equations, 27 (2011), no. 1, 1-8. 
[8] Tunç, C., Exponential stability to a neutral differential equation of first order with delay. Ann. Differential Equations 29 (2013), no. 3, 253-256.

[9] Tunç, C.; Sirma, A., Stability analysis of a class of generalized neutral equations, J. Comput. Anal. Appl., 12 (2010), no. 4, 754-759. 